DNA in plasma samples by applying the same PCR protocol as Drago et al, ${ }^{1,2}$ sequenced the amplification product, and could detect HHV-7 sequences in only about $50 \%$ of the samples, as opposed to $100 \%$ in the reports by Drago et al. The search for antibodies showed the absence of IgM antibodies, as well as no increase in IgG titer (except for 1 case), both of which are expected to increase in primary and/or reactivation infections.

(2) Yoshida ${ }^{6}$ applied the same nested PCR protocol as Drago et al ${ }^{1,2}$ to peripheral blood DNA of patients with PR and healthy individuals, and found a signal of equal intensity in all samples. This finding argues against an increase in viral DNA load in the PBMCs of patients with PR and against the specific occurrence of the viral sequences in the PR samples.

(3) Yasukawa et al amplified HHV-7 DNA from PBMCs in only 1 of 14 patients, failed to isolate HHV-7 from cocultures of PBMCs and SupT1 cells, and failed also to detect any increase in anti-HHV-7 IgG relative to normal control subjects.

In their letter above, Drago et al raise the interesting issue of whether an infectious agent, or markers thereof, must, or must not, be constantly present in an acute lesion induced by a herpesvirus. Both our knowledge on the biology of herpesvirus infections and the investigative tools available today have changed so much that there is currently a need to establish clear-cut criteria for pathogenetic association and causality. To review these criteria is beyond the purpose of the present reply. At the risk of oversimplifying the problem, in general, during acute phases of infections with herpesviruses (primary and reactivation), either 1 or both of the following conditions seem to apply: (1) the virus is present in the lesion and/or (2) there is an increase in the antibody response. At the current state of the art, in our opinion, the key issue concerning PR is whether the available evidence does, or does not, support an association with $\mathrm{HHV}-7$ infection or reactivation. Establishing a causative role should be a subsequent step. To establish an association, consistency of the association is a minimum requirement. Independent laboratories have failed to confirm the presence of HHV-7 in PR lesions, or to reveal serologic or immunologic markers of an active infection or reactivation in all patients with $P R$. At the current state of the art, even without taking into consideration our own study, it seems to us that the association between $\mathrm{HHV}-7$ and PR is not yet firmly established.

Werner Kempf, MD
Department of Dermatology
University Hospital Zurich
Gloriastrasse 31
CH-8092 Zurich, Switzerland
Volker Adams, MD
Leipzig, Germany
Guenter Burg, MD
Renato G. Panizzon, MD
Zurich
Gabriella Campadelli-Fiume, PhD
Bologna, Italy
Frank O. Nestle, MD
Zurich

1. Drago F, Ranieri E, Malaguti F, Losi E, Rebora A. Human herpesvirus 7 in pityriasis rosea [letter]. Lancet. 1997;349:1367.
2. Drago F, Ranieri E, Malaguti F, Battifoglio ML, Losi E, Rebora A. Human herpesvirus 7 in patients with pityriasis rosea. Dermatology. 1997;195 374-378.

3. Kempf W, Adams V, Kleinhans M, et al. Pityriasis rosea is not associated with human herpesvirus 7. Arch Dermatol. 1999;135:1070-1072.

4. Watanabe T, Sugaya M, Nakamura K, Tamaki K. Human herpesvirus 7 and pityriasis rosea [letter]. J Invest Dermatol. 1999;113:288-289.

5. Yasukawa M, Sada E, Machino H, Fujita S. Reactivation of human herpesvirus 6 in pityriasis rosea [letter]. Br J Dermatol. 1999;140:169.

6. Yoshida M. Detection of human herpesvirus 7 in patients with pityriasis rosea and healthy individuals [letter]. Dermatology. 1999;199:197.

7. Di Luca D, Zorzenon M, Mirandola P, Colle R, Botta GA, Cassai E. Human herpesvirus 6 and human herpesvirus 7 in chronic fatigue syndrome. J Clin Microbiol. 1995;33:1660-1661.

8. Kidd IM, Clark DA, Ait-Khaled M, Griffiths PD, Emery VC. Measurement of human herpesvirus 7 load in peripheral blood and saliva of healthy subjects by quantitative polymerase chain reaction. J Infect Dis. 1996;174:396-401.

9. Frenkel N, Schirmer EC, Wyatt LS, et al. Isolation of a new human herpesvirus from human CD4 T-cell. Proc Natl Acad Sci U S A. 1990;87:748-752.

10. Stefan A, De Lillo M, Frascaroli G, Secchiero P, Neipel F, CampadelliFiume G. Development of recombinant diagnostic reagents based on pp85 (U14) and p86(U11) proteins to detect the human immune response to human herpesvirus 7 infection. J Clin Microbiol. 1999;37:3980-3985.

11. Kempf W, Adams V, Wey N, et al. CD68 $8^{+}$cells of monocyte/macrophage lineage in the environment of AIDS-associated and classic-sporadic Kaposi's sarcoma are singly or doubly infected with human herpesviruses 7 and 6B. Proc Natl Acad Sci U S A. 1997;94:7600-7605

12. Kempf W, Adams V, Mirandola P, et al. Persistence of human herpesvirus 7 in normal tissues detected by expression of a structural antigen. J Infect Dis. 1998;178:841-845.

13. Berneman ZN, Ablashi DV, Li G, et al. Human herpesvirus 7 is a Tlymphotropic virus and is related to, but significantly different from, human herpesvirus 6 and human cytomegalovirus. Proc Natl Acad Sci U S A. 1992;89:10552-10556.

\section{Tumor Screening and Biology in Maliejant Melanomas}

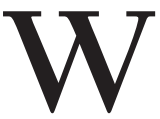

e read with interest the recent article and editorial that emphasize the increasing incidence of thin malignant melanomas (MMs). ${ }^{1,2}$ Similar results have been reported in different institutions, ${ }^{2}$ and that is certainly our experience as well. The analysis of those data highlights interrelated aspects of public health (screening) and tumor biology.

Although nondysplastic nevi have been reported to confer a small (2-fold) risk, clinically atypical (dysplastic) melanocytic nevi (AMN) confer substantial MM risk (2-fold for $1 \mathrm{AMN}$; 12-fold for $\geq 10 \mathrm{AMN}$ ). ${ }^{3}$ Based on this, clinicians can identify a population at high risk of MM for screening and intervention. Except for heritable MM, ${ }^{4}$ AMNs seem to represent a risk marker rather than a true precancerous lesion, ${ }^{3}$ especially for low-grade AMNs (unpublished data, 1999). The other risk factors for MM such as intermittent sun exposure are, on the one hand, less susceptible to intervention and, on the other hand, not significantly associated with an increased risk of mortality from MM. ${ }^{2}$ However, AMN diagnosis was controversial before the pathologist consensus convened by the World Health Organization Melanoma Programme $e^{5}$ explained the variable AMN incidence (Figure). The increased sensitivity for diagnosing early MM also determines the decreasing incidence of AMN with severe melanocytic dysplasia in the last few years (Figure), because many of those lesions are now classified as melanomas in situ. Therefore, the strong commitment of dermatologists and pathologists to the early diagnosis of MM to provide a better prognosis would partly explain this increase of MM incidence, as Berwick suggests. ${ }^{2}$ 


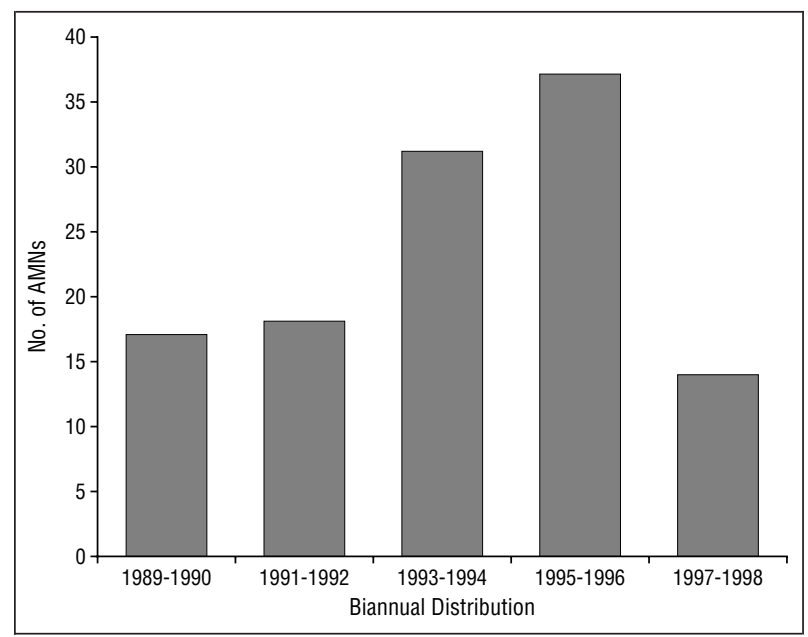

Distribution of atypical (dysplastic) melanocytic nevi (AMN) diagnosed between 1989 and 1998 at St Bartholomew's and the London National Health System Trust, London, England. The highest incidence was observed between 1993 and 1996 following the advent of the consensus on diagnostic criteria of AMN (published in 1991). ${ }^{5}$ The decreased incidence in 1997 and 1998 correlated with an increased rate of melanoma in situ.

Regarding MM biology, tumor thickness, apart from determining prognosis, identifies 2 biologically different types of MM. This is not specific for MM, but applies generally to tumor pathobiology. Evidence from other organ systems including the uterine cervix (squamous cell carcinoma), endometrium or stomach (adenocarcinoma), and urinary bladder (transitional cell carcinoma) reveals the same 2 main types of malignancies. In general, most tumor risk factors and true precancerous lesions that are susceptible to screening programs are mainly associated with malignancies of protracted natural history, which explains the unchanged incidence of high-grade neoplasms in those organs. These highgrade tumors normally develop de novo and show no associated precancerous lesions, as expressed in thick MMs that are frequently nodular and mostly fast-growing tumors. This sort of tumor is not susceptible to any screening, which explains the failure of efficient early detection programs. ${ }^{1}$ This equally answers the open question that is the editorial's title ${ }^{2}$ : no current screening program will effectively address the early diagnosis of thick nodular MM in a potentially curable stage. Unfortunately, our technology does not yet offer this possibility. Only the advent of new diagnostic techniques to detect subtle genetic or functional changes of early transformed melanocytes can offer a better prognosis for patients with nodular MM.

Lucia Pozo, MD, PhD

Salvador J. Diaz-Cano, MD, PhD

Department of Histopathology $\mathcal{E}$ Morbid Anatomy

St Bartholomew's and the London National

Health System Trust

Whitechapel

London E1 1BB, England

(e-mail:s.j.diaz-cano@mds.qmw.ac.uk)

1. Lipsker DM, Hedelin G, Heid E, Grosshans EM, Cribier BJ. Striking increase of thin melanomas contrasts with stable incidence of thick melanomas. Arch Dermatol. 1999;135:1451-1456.
2. Berwick M. Why are people still dying from melanoma? Arch Dermatol. 1999; 135:1534-1536.

3. Tucker MA, Halpern A, Holly EA, et al. Clinically recognized dysplastic nevi: a central risk factor for cutaneous melanoma. JAMA. 1997;277:1439-1444.

4. Clark WH Jr, Reimer RR, Greene M, Ainsworth AM, Mastrangelo MJ. Origin of familial malignant melanomas from heritable melanocytic lesions: "the B-K mole syndrome." Arch Dermatol. 1978;114:732-738.

5. Clemente C, Cochran AJ, Elder DE, et al. Histopathologic diagnosis of dysplastic nevi: concordance among pathologists convened by the World Health Organization Melanoma Programme. Hum Pathol. 1991;22:313-319.

\section{An Alternate Explanation for the Increase in the Incidence of Melanoma Being Restricted to Patients With Thin Lesions}

D rs Lipsker et $\mathrm{al}^{1}$ recently reported that in a carefully conducted population-based study there was a dichotomy between the rising incidence of thin melanomas and the stable incidence of thicker lesions. The authors point out that the excision of increasing numbers of thin melanomas has had no effect on the incidence of thick melanomas. They propose as an explanation that thin and thick melanomas have different epidemiological features, and that the increased incidence of this cancer is owing to the recognition of a thin form of melanoma that is innocuous and unlikely to cause death if not treated.

However, there is an alternate interpretation that leads to a different conclusion. It flows from a simple mathematical analysis of the differing impacts of (1) the increasing incidence of melanoma, and (2) early detection on the proportion of patients who will have thin as opposed to thick lesions at diagnosis. It is illustrated by the following example: Assume that at baseline there are 100 new melanomas per year in a given population, and that $50 \%$ of these are diagnosed while still thin; 50 thin melanomas will then be diagnosed per year. Suppose that at a later date the incidence of melanoma has doubled, but that as a result of earlier detection the proportion diagnosed while still thin improves by half to $75 \%$. The incidence of melanoma will now be 200 new cases per year, $75 \%$ (150) of which will be thin, and the number of thick melanomas will remain at 50 per year. Thus, a doubling in the incidence of melanoma coupled with a 50\% improvement in early diagnosis results in a tripling of cases diagnosed while still thin, with no changes in the number of thick lesions.

The data presented by Drs Lipsker et $\mathrm{al}^{1}$ is remarkably consistent with this interpretation. The number of new melanomas in Strasbourg, France, more than doubled between 1980 and 1997, from fewer than 40 per year to more than 90 per year (see their Figure 2). During the same time the number of melanomas diagnosed while still thin increased 4-fold from less than 15 per year to approximately 60 per year (see their Figures 6 and 7), while that of thick melanomas increased only slightly.

Are these different interpretations of more than academic importance? Yes, because their implications are very different. That of Drs Lipsker and colleagues ${ }^{1}$ implies that the effort to reduce melanoma mortality by early detection and treatment is ineffective because the lesions being detected and removed would not have caused death in any event. By contrast, the interpretation pro- 
posed here implies that this effort is effective, as evidenced by the increasing proportion of melanomas diagnosed while still thin and at a stage when they are completely curable. The fact that the number of thick melanomas is not decreasing reflects that the incidence of this cancer is increasing more rapidly than our ability to detect it early. The implication is that efforts at early diagnosis should be intensified and coupled with an equally strong effort to reduce the incidence of this cancer.

Jean-Claude Bystryn, MD

Department of Dermatology

New York University Medical Center

550 First Ave

New York, NY 10016

1. Lipsker DM, Hedelin G, Heid E, Grosshans EM, Cribier BJ. Striking increase of thin melanomas contrasts with stable incidence of thick melanomas. Arch Dermatol. 1999;135:1451-1456.

\section{In reply}

We thank Dr Bystryn for his interest in our recently published work in this journal. ${ }^{1}$ Dr Bystryn proposes an alternate explanation for the increase in the incidence of thin melanomas. His explanation relies on a mathematical analysis of the differing impacts of increasing incidence and early detection. According to his model, the combined effect of an overall increase in melanomas and simultaneous improvement in early detection of thin lesions could lead to a stable number of thick melanomas (because their proportion decreases) and an increase in the number of thin melanomas (the proportion of which increases). We agree that the increase in incidence of thin melanomas is in part related to better and earlier detection of melanomas. We also agree that early detection is the best treatment of melanoma and remains essential.

However, we do not think that this mathematical analysis explains the difference we observed between incidence rates of thick and thin melanomas. Indeed, this model implies 3 postulates, which Dr Bystryn did not address. First, it supposes that every thick melanoma is the result of a thin melanoma. Second, it supposes that the time lapse during which a thin lesion becomes a thick lesion is long enough to allow efficient detection at the thin stage in all cases. That would mean that this time is compatible with detection and that people would consult with a physician during this period. Third, it supposes that the whole population would be screened. If any of these postulates would not hold true, improvement of detection alone could not explain the stability in incidence of thick melanomas while there is a steady increase in thin melanomas.

Our data support the notion that the lapse of time during which thin tumors become thick tumors is not always long enough to allow detection. Indeed, this time lapse can be very short and was less than 3 months in some patients with thick, fast-growing, nodular melanomas. Furthermore, our data showed a constant and regular increase in the incidence of thin melanomas over 18 years, while the incidence of thick melanomas remained stable during this period. According to Dr Bystryn's model, this would mean that there was a proportional, year-by-year improvement of detection during this period. However, until 1998 there was no organized detection of melanoma in the Bas-Rhin region of France. Furthermore, we showed in a Strasbourg University Hospital-based study ${ }^{2}$ that the proportion of melanomas diagnosed by means of detection was extremely low until the 1990s, while incidence of thin melanomas had already doubled by this time compared with 1980. Even though detection is essential, its efficiency, eg, the percentage of the population that is actually screened, probably remains low in the Bas-Rhin and can still be improved. Thus, it probably does not explain why incidence rates of thick tumors remained stable during this period. Therefore, and although the mathematical analysis suggested by Dr Bystryn may account for part of the increase in thin melanomas, further studies should be conducted to clarify why the evolution of incidence rates of thick and thin melanomas do not seem to be related.

Dan M. Lipsker, MD

Laboratoire d'Histopathologie Cutanée Clinique Dermatologique

Hôpitaux Universitaries de Strasbourg Strasbourg, France

Guy Hedelin, PhD

Strasbourg

1. Lipsker DM, Hedelin G, Heid E, Grosshans EM, Cribier BJ. Striking increase of thin melanomas contrasts with stable incidence of thick melanomas. Arch Dermatol. 1999;135:1451-1456.

2. Lipsker D, Heid E, Grosshans E, Cribier B. Le mélanome au CHU de Strasbourg: étude sur 30 ans. Ann Dermatol Venereol. 1998;125:241-246.

\section{VIGNETTES}

\section{Treatment of Labial Lentigos in Atopic Dermatitis With the Frequency-Doubled Q-Switched Nd:YAC Laser}

$\mathbf{R}$ ecently, atopic dermatitis has been increasing throughout the world, and there have been many labial lentigos caused by a postinflammation of atopic dermatitis. ${ }^{1}$ Laser therapy, such as the normal ruby laser $^{2}$ and the Q-switched ruby laser, ${ }^{3}$ is one of the treatment methods. We make the first report of 4 cases of labial lentigos in atopic dermatitis treated with the frequency-doubled Q-switched Nd:YAG laser at a wavelength of $532 \mathrm{~nm}$. We achieved rapid results and a dramatic clearing of the lesions without cutaneous alterations in skin texture.

Patients and Methods. We used the frequency-doubled Q-switched Nd:YAG laser (Continuum Biomedical Inc, Livermore, Calif), which has a pulse duration of 5 to 7 nanoseconds, a wavelength of $532 \mathrm{~nm}$, a pulse repetition rate of $10 \mathrm{~Hz}$, and a spot size of $3 \mathrm{~mm}$. Local anesthesia using 60\% lidocaine tape was placed on the affected part 1 hour before laser irradiation. After laser irradiation, $0.12 \%$ betamethasone valerate ointment containing $0.1 \%$ gentamicin sulfate was applied for 3 days. All subjects had labial lentigos with atopic dermatitis. No 